\title{
MET NM_000245.3:c.3209T>A
}

National Cancer Institute

\section{Source}

National Cancer Institute. MET NM 000245.3:C.3209T>A. NCI Thesaurus. Code C162298.

A nucleotide substitution at position 3209 of the coding sequence of the MET gene where thymine has been mutated to adenine. 